\title{
Sharing Cost of Shared Services Centre ${ }^{\#}$
}

\author{
Tomáš BUUS
}

\section{Introduction}

As far as we know pricing of intra-company or intra-group deliveries is issue having ties with efficient resource allocation, managers' motivation, and ability to defend the chosen approach against revenue authority. Unfortunately there is a lot of literature regarding the inventory (cash) optimization models, route optimization, batch size optimization, etc., with respect to a very various specification of cost functions, but very little literature, which deals with prices set by centres, which provide these services. These can include delivery costs, customer loss implied cost, as well as storage cost. The problem of pricing of the services provided by shared services centre is on the border between operational research, taxation and financial management. Curiously a little attention is paid to it as we have found no scholar paper that would directly deal with this problem, except for those dealing with capacity sizing and pricing in the IT industry (e.g. Maglaras and Zeevi, 2003) or airports (e.g. Zhang and Zhang, 2010). Some attempts to find a solution to pricing of shared services was made by Buus and Žd'árek (2008), but without any large success.

We develop tiny general model of apportionment of cost generated by creation and maintaining capacity in shared services centre (SSC) in this paper.

The rest of this paper is organized as follows:

1. description of shared services centre costs genesis,

2. model development,

\# This paper was prepared in the framework of research plan Development of Accounting and Financial Theory and its Application in Practice from Interdisciplinary Point of View (registered number MSM 6138439903).

* Ing. Tomáš Buus, Ph.D. - Assistant Professor; Department of Corporate Finance and Valuation, Faculty of Finance and Accounting, University of Economics, Prague, W. Churchill Sq. 4, 13067 Prague 3, Czech Republic; <buust@vse.cz>. 
3. conclusions and discussion.

\section{Cost of Shared Services Centre}

Maybe the best way to describe shared services centre cost is an example. In SSC the costs are generated both by the necessary capacity to perform the functions in the time and at the place needed. On the other hand a sort of cost that is generated by the functioning itself, that would not emerge without use of the created capacity. The proportion of each of the types of cost depends upon the nature of SSC.

A very example of SSC, which generates a large portion of capacity cost, is a distribution centre. Either built or rented, the more space distribution centre needs, the higher cost, although the turnover can be still the same. The cost generated by turnover in distribution centre is likely to be the personnel cost, because if flow of goods is higher, we need more personnel to handle it. On the other hand rent (or economical rent), lighting, heating and overhead costs are those, which are largely independent from the quantity of goods that go through distribution centre, but rather depend on the ability of distribution centre to keep the stock of goods as low as possible.

An SSC, which is in some manner similar to distribution centre, but very different in other aspects, is technical support (or servicing). Company needs to keep qualified staff in high enough numbers to handle the requirements, because the cost of delay in production or customer dissatisfaction in case he/she needs the technical support, is much larger than cost of keeping the necessary staff. Contrary to the distribution centre, the flow of requirements is not easy to influence.

The opposite type of SSC, which bears low portion of capacity cost, is cash pooling and/or netting centre. Here the capacity is represented by the ability to decrease the net working capital, to decrease the need of the whole company (or multibusiness enterprise) for cash. Quite large part of financial costs associated with cash management is represented by bank fees. Moreover the capacity costs in the cash pool/netting centre are not so easily distinguishable from the costs borne by operation of the services centre, i.e. by necessity to fulfil the service requirements as both of these types of costs can take a form of overhead cost as well as interest. 


\section{Capacity Cost Model}

In case of stochastic models of inventory management as well as for stochastic models of short-term financial assets management the expected average levels of cash balance or inventory can be in some basic cases (e.g. Miller - Orr, 1966 or Frenkel - Jovanovic, 1980) described or approximated by function

$$
L_{P}=f\left(\stackrel{+}{\mu_{P}},{\stackrel{+}{\sigma_{P}}}^{2}\right)
$$

where $\mu \in R^{+}$is the mean value of absolute values of flow of cash or inventory from and into SSC (i.e. drift), $\sigma \in R^{+}$is standard deviation of that flow and lower index $P$ denotes portfolio of all cash flows from (to) the SSC (for flows attributable to particular company $j$ we would use lower index $j$ ). In fact this form of average (and peak) optimal level of inventory is inherent to models with delivery size uncertainty and delivery disruption (cp. Schmitt and Snyder, 2012) if the supplier's failures follow the Bernoulli distribution.

By definition $\mu$ is not function of $\sigma$ and $\sigma$ is not function of $\mu$. Finally let us note that $f$ does not have to be homogenous of $1^{\text {st }}$ degree nor for $\mu_{P}$, nor for $\sigma_{P}^{2}$, i.e. it is possible that finally something like $\sigma_{p}^{4 / 3}$ appears in the $f$ function, which is still function of $\sigma_{P}^{2}: \sigma_{p}^{4 / 3}=\left(\sigma_{p}^{2}\right)^{2 / 3}$. The notation $f\left(\stackrel{+}{\mu}_{P}^{+}, \sigma_{P}^{2}\right)$ means that the function is strictly growing in both parameters. Our model further assumes that the unit cost of both parts of $L_{P}$, i.e. $\mu_{P}$ and $\sigma_{P}^{2}$, are constant with respect to $\mu_{P}$ and $\sigma_{P}^{2}$.

The design described in (1) captures most of the stochastic inventory (cash) management system optimization solutions, even some of the newest ones, like (Indenfurth - Vogelgesang, 2011). Evidently SSC needs not stock to fulfil a time-varying continuous requirements of customers, as the retail store does, but its service request flow has rather a batch nature (especially in the case of distribution centre). 
If we allowed for some constant cost of capacity, independent from variability of flows from (to) SSC, the total cost of capacity invoked by variability of flows $\left(C_{\sigma}\right)$ would be for any company in multibusiness enterprise ("MBE"), but also for the whole SSC

$$
\begin{aligned}
& C_{P}=C-C_{0}, \\
& C_{P}=C_{\sigma}+C_{\mu}
\end{aligned}
$$

where $C_{0}$ is cost that is constant with respect to both $\sigma$ and $\mu, C_{\mu}$ is part of costs, generated by flow. We have further split the cost of servicing the portfolio of requirements $C$ into cost generated by variability and by the mean of the requirements flow.

Let us remind how the derivatives of $f$ were computed if any flow from (to) SSC increased by very small part of that flow $d:^{1}$

$$
\begin{aligned}
& \quad \frac{\partial f\left(\mu_{P}, \sigma_{P}^{2}\right)}{\partial \sigma_{P}} \cdot d \sigma_{P}=\frac{\partial f\left(\mu_{P}, \sigma_{P}^{2}\right)}{\partial \sigma_{P}^{2}} \cdot \frac{\partial \sigma_{P}^{2}}{\partial \sigma_{P}} \cdot d \sigma_{P}= \\
& =\frac{\partial f\left(\mu_{P}, \sigma_{P}^{2}\right)}{\partial \sigma_{P}^{2}} \cdot \lim _{d \rightarrow 0} \frac{(1+d)^{2} \cdot \sigma_{P}^{2}-\sigma_{P}^{2}}{d \sigma_{P}} \cdot d \sigma_{P}=, \\
& =\frac{\partial f\left(\mu_{P}, \sigma_{P}^{2}\right)}{\partial \sigma_{P}^{2}} \cdot \lim _{d \rightarrow 0}\left[(1+d)^{2} \cdot \sigma_{P}^{2}-\sigma_{P}^{2}\right]
\end{aligned}
$$

and if any flow from (to) SSC that arises because of company $j$ increased by very small part of that flow $d$ :

\footnotetext{
1 It is obvious that $\sigma_{(1+d) \cdot x}=(1+d) \cdot \sigma_{x}$.
} 


$$
\begin{aligned}
& \frac{\partial f\left(\mu_{P}, \sigma_{P}^{2}\right)}{\partial \sigma_{j}} \cdot d \sigma_{j}=\frac{\partial f\left(\mu_{P}, \sigma_{P}^{2}\right)}{\partial \sigma_{P}^{2}} \cdot \frac{\partial \sigma_{P}^{2}}{\partial \sigma_{j}} \cdot d \sigma_{j}= \\
& =\frac{\partial f\left(\mu_{P}, \sigma_{P}^{2}\right)}{\partial \sigma_{P}^{2}} \cdot \lim _{d \rightarrow 0} \frac{(1+d)^{2} \cdot \sigma_{P}^{2}-\sigma_{P}^{2}}{d \sigma_{j}} \cdot d \sigma_{j}=, \\
& =\frac{\partial f\left(\mu_{P}, \sigma_{P}^{2}\right)}{\partial \sigma_{P}^{2}} \cdot \lim _{d \rightarrow 0}\left[(1+d)^{2} \cdot \sigma_{P}^{2}-\sigma_{P}^{2}\right]
\end{aligned}
$$

but in the case of increasing the flow of $j^{\text {th }}$ company the limit would be in equation (4)

$$
\begin{aligned}
& \left.\lim _{d \rightarrow 0} \mid(1+d)^{2} \cdot \sigma_{P}^{2}-\sigma_{P}^{2}\right\rfloor= \\
& =\sigma_{P}^{2}+2 \cdot d \cdot \operatorname{cov}_{P, j}+d^{2} \cdot \sigma_{j}^{2}-\sigma_{P}^{2} \approx 2 \cdot d \cdot \operatorname{cov}_{P, j}
\end{aligned}
$$

contrary to the value of limit in equation (3), i.e. for increase of the magnitude of flows of whole SSC

$$
\begin{aligned}
& \lim _{d \rightarrow 0}\left[(1+d)^{2} \cdot \sigma_{P}^{2}-\sigma_{P}^{2}\right]= \\
& =\left(1+2 \cdot d+d^{2}\right) \cdot \sigma_{P}^{2}-\sigma_{P}^{2} \approx 2 \cdot d \cdot \sigma_{P}^{2}
\end{aligned}
$$

because higher powers of $d$ are negligibly small

$$
\underset{n>1 ; n \in N}{\forall}\left(\lim _{d \rightarrow 0} \frac{d^{n+\phi}}{d^{\phi}}=0\right) ; \phi \in R^{+} .
$$

Reflecting (5) into (4) and (6) into (3) we get

$$
\frac{\partial f\left(\mu_{P}, \sigma_{P}^{2}\right)}{\partial \sigma_{P}} \cdot d \sigma_{P}=\frac{\partial f\left(\mu_{P}, \sigma_{P}^{2}\right)}{\partial \sigma_{P}^{2}} \cdot 2 \cdot d \cdot \sigma_{P}^{2},
$$

and 


$$
\frac{\partial f\left(\mu_{P}, \sigma_{P}^{2}\right)}{\partial \sigma_{j}} \cdot d \sigma_{j}=\frac{\partial f\left(\mu_{P}, \sigma_{P}^{2}\right)}{\partial \sigma_{P}^{2}} \cdot 2 \cdot d \cdot \operatorname{cov}_{P, j}
$$

We have required the unit price of variability to be the same. It is natural because otherwise we would have problem to split the cost of flow variability generated by all companies using SSC services. Therefore increases of unit cost in proportion to increases of variability are the same if invoked by addition of risk by $j^{\text {th }}$ or in portfolio in general:

$$
\frac{d C_{\sigma, j}}{\frac{\partial f\left(\mu_{P}, \sigma_{P}^{2}\right)}{\partial \sigma_{j}} \cdot d \sigma_{j}}=\frac{d C_{\sigma, P}}{\frac{\partial f\left(\mu_{P}, \sigma_{P}^{2}\right)}{\partial \sigma_{P}} \cdot d \sigma_{P}}
$$

so that

$$
d C_{\sigma, j}=d C_{\sigma, P} \cdot \frac{\frac{\partial f\left(\mu_{P}, \sigma_{P}^{2}\right)}{\partial \sigma_{P}^{2}} \cdot 2 \cdot d \cdot \operatorname{cov}_{P, j}}{\frac{\partial f\left(\mu_{P}, \sigma_{P}^{2}\right)}{\partial \sigma_{P}^{2}} \cdot 2 \cdot d \cdot \sigma_{P}^{2}}
$$

and after some rearrangement

$$
d C_{\sigma, j}=d C_{\sigma, P} \cdot \frac{\operatorname{cov}_{P, j}}{\sigma_{P}^{2}} .
$$

We can see that because (2) holds for any group of companies within the SSC and because of a part of cost being constant with respect to variability, the relation between cost borne by all $n$ companies within SSC and cost borne by the whole portfolio $P$ of them is

$$
\sum_{j=1}^{n}\left[\frac{\operatorname{cov}_{P, j}}{\sigma_{P}^{2}} \cdot C_{\sigma, P}\right]=C_{\sigma, P},
$$

as sum of all covariances within portfolio measured in monetary or physical units equals to variance of that portfolio 


$$
\sum_{j=1}^{n} \operatorname{cov}_{P, j}=\sigma_{P}^{2} .
$$

It is trivial to see that the customers of SSC should bear its capacity cost by the share they generate it, or in other words, the costs that emerge because of inability of SSC customers to coordinate their orders with orders of the other SSC customers and with the deliveries.

This way we have derived solution for e.g. cash pool or netting center as in the cash pool or netting center the end-of day balance for members of multibusiness enterprise (hereinafter "MBE") is a given amount, usually zero. They pay and get paid, in fact, on the expense (in favor) of cash pool. However there could be SSC, which needs to balance its needs with the rest of the MBE. Distribution center can be an example again.

Now let us assume that each of the $n$ companies, which use the services, provided by SSC, needs some capacity on their own. The amount by which that company increases the need for capacity by SSC is equal to $\operatorname{cov}_{P, j}$ as described by (14). The function generating capacity requirement at the company itself can be described by (1) as well as at the SSC as the company (customer of SSC) faces similar cost functions. Some part of the function is generated by capacity requirement, some by revenue loss if capacity is insufficient, some part by transaction (delivery) cost. We can then modify any of the known functions of stock management cost (including the opportunity cost) by adding the cost borne by SSC as a cause of $j$-th customer requirements. As the capacity cost function of SSC becomes a part of cost function of each customer, they will then also by deciding to minimize their cost functions also leveloff the cost of their own capacity, inability to serve customer (if there was insufficient capacity), transaction cost (which tend to be constant per transaction) and finally the cost borne by SSC.

\section{Cost generated by flow}

In the previous chapter we have addressed the part of cost generated by flow variability. It is however evident that even some part of capacity is not dependent on the smoothness of the order (both by SSC and by SSC customers) flow. An example can be number of ramps in the distribution 
center. The driver of this variable is expected flow from/to the SSC. Another example is

1. number of staff and equipment needed to handle the customers' requirements in the distribution center (again), or

2. per payment fee in the cash pool,

3. or even in the SSC, its size is totally driven by capacity, e.g. in the technical support center, a part of personnel cost, that is driven by the number of customer requirements satisfied.

In the same way we have derived the optimal solution in the case of capacity cost generated by variance of requirement flow $\sigma_{P}^{2}$, we can do the derivation in the case of cost generated by the average level of flow $\mu_{P}$ in SSC.

$$
d C_{\mu, j}=d C_{\mu, P} \cdot \frac{\mu_{P}}{\mu_{j}} .
$$

\section{Conclusions and discussion}

In the presented paper we develop model of apportionment of cost generated by variability and mean value of flows from (to) shared services centre. It can be either cash pool or distribution centre, or even some kind of customer service centre. The apportionment formula for the cost of capacity generated by flow variability turns out to be regression coefficient of flow to (from) the distribution centre (cash pool) generated by particular company within the multibusiness enterprise as endogenous variable to flow of inventory (cash) for the whole distribution centre (cash pool) as exogenous variable. The cost generated by the flow of requirements (goods, money) itself, i.e. by the mean value of the flow, has to be split between SSC customers according to their share on that flow. Result does not depend on the form of variability cost function.

An advantage of our solution is that it is self-regulating as far as the assumptions are fulfilled (see also below) and no central policy is needed. Our solution brings the free market into the area that has been dominated by centralized decision-making processes.

There is however a number of practical issues that SSC needs to address in practice. 
The information system (IS) that would provide the information necessary to optimize the flow from/to the SSC is an issue itself. Luckily, in the time of internet and/or secured tunnel connections almost everywhere it is just a technological problem. However, we have to mention that the ability of SSC to communicate the inflow/outflow schedule with its counterparts is a crucial prerequisite to the functionality of our model.

If the customers of SSC have available information about the schedule of deliveries from the SSC and to the SSC (the inflow can be considered in case of distribution centre or cash pool/netting centre) then they have tool to optimize their delivery requirements accordingly to minimize $\sigma_{P}^{2}$ and $\mu_{j}$. But the practice is not so straightforward so that some export/import limitations may be faced in case of SSC providing its services internationally. Furthermore there could be objections to MBE members to keep to their orders so that they might need to cancel them, etc. A penalization that covers the cost has to be in play then. It is mostly the case of cash pool/netting center, where a need to address the issues of mutual lending/borrowing is of special importance. The interest rates as well as exchange rates (if netting is realized internationally) need to be assessed according to the risks borne by all sides of transaction, with regard to the fact that these transactions are conducted between related parties, so that the risks are not the same as at unrelated transactions.

\section{References}

[1] Frenkel, J. A. - Jovanovic, B. (1980): On Transactions and Precautionary Demand for Money. Quarterly Journal of Economics, 1980, vol. 95, no. 1, pp. 25-43.

[2] Indenfurth, K. - Vogelgesang, S. (2011): Concepts for Safety Stock Determination under Stochastic Demand and Different Types of Random Production Yield. [on-line], Magdeburg, Otto von Guericke Universität Magdeburg, c2011, [cit. 10 ${ }^{\text {th }}$ October, 2011], <http://www.fww.ovgu.de/fww_media/femm/femm_2011/2011_03.p df $>$

[3] Maglaras, C. - Zeevi, A. (2003): Pricing and Capacity Sizing for Systems with Shared Resources: Approximate Solutions and Scaling Relations. Management Science, 2003, vol. 49 no. 8, pp. 1018-1038. 
[4] Miller, H. M. - Orr, D. (1968): A Model of the Demand for Money by Firms. Quarterly Journal of Economics, 1966, vol. 80, no. 3, pp. 413435.

[5] Buus, T. - Žd'árek, V. (2008): Transfer Prices for Management of Current Assets and Liabilities. (in Czech: Transferové ceny při řizení krátkodobých aktiv a pasiv $v$ koncernu). Praha, University of Economics, 2008.

[6] Schmitt, A. J. - Snyder, L. V. (2012): Infinite-horizon models for inventory control under yield uncertainty and disruptions. Computers \& Operations Research, 2012, vol. 39, no. 4, April 2012, pp. 850-862.

[7] Zhang, A. - Zhang, Y. (2010): Airport capacity and congestion pricing with both aeronautical and commercial operations. Transportation Research Part B: Methodological, 2010, vol. 44, no. 3, pp. 404-413. 


\title{
Sharing Cost of Shared Services Centre
}

\author{
Tomáš BUUS
}

\begin{abstract}
In the presented paper we develop model of apportionment of cost generated by variability and mean value of flows from (to) shared services centre. It can be either cash pool or distribution centre, or even some kind of customer service centre. The apportionment formula for the cost of capacity generated by flow variability turns out to be regression coefficient of flow to (from) the distribution centre (cash pool) generated by particular company within the multibusiness enterprise as endogenous variable to flow of inventory (cash) for the whole distribution centre (cash pool) as exogenous variable. The cost generated by the flow of requirements (goods, money) itself, i.e. by the mean value of the flow, has to be split between SSC customers according to their share on that flow. Result does not depend on the form of cost function as long as it is strictly increasing function of flow from (into) SSC (orders, stock, cash) and of mean of that flow.
\end{abstract}

Key words: Distribution centre; Cash Pool; Capacity; Cost Attribution.

JEL classification: C61, G39, M21. 